\title{
Parámetros poblacionales de la rana dorada, Phyllobates terribilis (Myers et al., 1978) (Dendrobatidae), en una localidad de Buenaventura, Valle del Cauca, Colombia
}

\author{
Population parameters of the Golden Poison Frog, Phyllobates terribilis (Myers et \\ al., 1978) (Dendrobatidae), in a location of Buenaventura, Valle del Cauca, \\ Colombia
}

\section{Stefhania Alzate-Lozano, Rodrigo Lozano-Osorio y Carlos Galvis}

\section{Resumen}

El objetivo de este estudio fue estimar el tamaño, densidad, probabilidad de supervivencia $(\varphi)$, de detección $(p)$, y describir las condiciones del hábitat de una población de la rana dorada (Phyllobates terribilis, Dendrobatidae) en bajos del río Naya, Buenaventura, Colombia. Se muestrearon 3 parcelas con área de $1000 \mathrm{~m}^{2}$ cada una en enero del 2015. Los datos obtenidos se analizaron con el modelo Jolly-Seber, usando la parametrización de POPAN. El tamaño poblacional fue 32,5 $\pm 4,3$ ind. y la densidad 0,011 ind $/ \mathrm{m}^{2}$. Las probabilidades de $\varphi(0,74 \pm 0,08)$ y $p(0,56 \pm 0,13)$ fueron constantes en todas las parcelas. La probabilidad de supervivencia y detección poblacional fueron altos en este lugar y periodo de estudio. La densidad y el tamaño poblacional calculados pueden tener mayor relación con la disponibilidad de recursos o con condiciones microclimáticas y no con la modificación del hábitat, debido a que esta población presenta niveles de tolerancia a ambientes con algún grado de modificación. Las altas probabilidades de $\varphi$ y $p$, pueden estar asociadas con la duración del muestreo, hábitos territoriales, fidelidad de hábitat, baja depredación, aposematismo y tamaño corporal. Esta información será útil para planear medidas de conservación de esta especie endémica de Colombia.

Palabras clave. Captura-Recaptura. Densidad. Hábitat. Perturbación. Supervivencia.

\begin{abstract}
The purpose of this study was to estimate the size, density, probability of survival $(\varphi)$ and detection $(p)$ and habitat conditions of a population of Golden Poison Frog (Phyllobates terribilis, Dendrobatidae) of Naya River, Buenaventura, Colombia. Three plots of $1000 \mathrm{~m}^{2}$ were sampled four times in January, 2015. Population data were analyzed through the Jolly-Seber model, using POPAN parameterization. Results indicate population size of $32.5 \pm 4.3$ ind. and population density of $0.011 \mathrm{ind} / \mathrm{m}^{2}$. Values of $\varphi$ and $p$ were constant between plots; $\varphi$ equaled $0.74 \pm 0.08$, and $p 0.56 \pm 0.13$. The probability of survival and detection of this population are high in the location and period of study. Calculated density and population size may have a greater relation to resource availability or microclimatic conditions than to habitat modification because this population has levels of tolerance to environments with some degree of modification. High $\varphi$ and $p$ values may be associated with the duration of sampling, territorial habits,
\end{abstract}


low predation, aposematism, and body size. The population of $P$. terribilis that was studied tolerates different environments with some degree of modification. This information will be useful to improve the conservation measures of this species, which is endemic to Colombia.

Keywords. Capture-recapture. Density. Disturbance. Habitat. Survival.

\section{Introducción}

Los anfibios han sido uno de los grupos más amenazados a nivel mundial, debido a múltiples factores como la pérdida y fragmentación de hábitats, enfermedades infecciosas, uso de pesticidas agrícolas, cambio climático, especies invasoras, comercio ilegal, contaminación ambiental y los efectos sinérgicos de todos estos factores (Young et al., 2001; Young et al., 2004; Pounds et al., 2006). Según la lista roja de la Unión Internacional para la Conservación de la Naturaleza (UICN, www. iucnredlist.org), más del $30 \%$ de los anuros a nivel mundial se encuentran en alguna categoría de peligro, convirtiéndolos en uno de los órdenes con mayor número de especies amenazadas.

En Latinoamérica, al menos 13 países han experimentado disminuciones poblacionales $\mathrm{o}$ extinciones de anfibios en los últimos 20 años, afectando a 30 géneros y 9 familias entre las cuales se incluye Dendrobatidae (Young et al., 2001). En Colombia, el 25 \% de las especies de esta familia analizadas por la UICN, están en alguna categoría de amenaza, mientras que el 32 \% tiene datos deficientes (DD).

La rana dorada (Phyllobates terribilis Myers et al., 1978) es una especie endémica de Colombia (Figura 1), está en la categoría En Peligro Crítico (CR) a nivel nacional, de acuerdo con el libro rojo de los anfibios de Colombia (CastroHerrera, 2004) y la resolución 0192 de 2014 del Ministerio de Ambiente y Desarrollo Sostenible (MADS). A nivel internacional, según la UICN, $P$. terribilis se encuentra categorizada como En Peligro (EN) (UICN). Esta especie pertenece a la familia Dendrobatidae y es reconocida por su comportamiento complejo, el cual incluye cuidado parental y alta territorialidad, además de coloración aposemática en algunos de sus géneros (Santos et al., 2003; Grant et al., 2006 ) y producción de sustancias tóxicas con alta importancia farmacológica en su cuerpo (Pennisi, 1992; Saporito et al., 2004; Berman, 2004). P. terribilis es una de las especies más venenosas de esta familia, siendo al menos veinte veces más toxica que otras ranas venenosas (Myers et al., 1978; Pröhl, 2005). Esta especie se puede distinguir de casi todas las especies de dendrobátidos por su mayor tamaño (37 y $45 \mathrm{~mm}$ en machos y 40-47mm en hembras), coloración dorsal brillante homogénea amarilla, naranja/dorada o crema verdosa (Myers et al., 1978; Lötters et al., 1997), y mayor toxicidad (Myers et al., 1978). En cuanto a sus características ecológicas, solo se ha reportado la presencia en la hojarasca de bosques primarios o bosques poco perturbados de la cordillera Occidental, en los departamentos de Cauca y Valle del Cauca de Colombia (Myers et al., 1978; Ruiz- Carranza et al., 1996; Castro-Herrera, 2004; Márquez et al., 2012). En términos culturales, es importante debido a que sus toxinas han sido usadas por comunidades indígenas Emberá para cazar (Myers et al., 1978).

En Colombia, los estudios acerca de la evaluación de características poblacionales de la familia Dendrobatidae son pocos, y aún menos los que utilizan técnicas de Captura-Marcaje-Recaptura (CMR) (Loaiza-Piedrahíta et al., 2016). Por otra parte, los estudios se han centrado principalmente en la región Andina y solo uno se ha realizado en la región Pacífica (LozanoOsorio, 2015). De estos estudios, dos realizaron estimaciones de densidades poblacionales (Marín y Gómez-Hoyos, 2011; Ríos et al., 2011) y otros tres usan el método de CMR para calcular algunos parámetros poblacionales como tamaños, 
probabilidades de supervivencia y de detección, entre otros (Molina-Zuluaga et al., 2014; LozanoOsorio, 2015; Loaiza-Piedrahíta et al., 2016).

El conocimiento de las características poblacionales de los anfibios es crucial para la conservación de su diversidad (Vitt y Caldwell, 2009). Sin embargo, son pocos los estudios sobre los estados $\mathrm{y}$ tendencias poblacionales. En consecuencia, se desconocen las causas de las disminuciones, los declives futuros y cómo se restablecen las poblaciones. Esto dificulta la implementación de medidas de conservación adecuadas (Lips et al., 2001; Funk et al., 2003; Blaustein et al., 2011). Por lo tanto, y teniendo en cuenta el estado actual de conservación de esta especie, esta investigación tiene como propósito estimar parámetros poblacionales como la probabilidad de detección, el tamaño y la densidad, y describir las características generales del hábitat de una población de $P$. terribilis en el resguardo indígena Joaquincito, Valle del Cauca.
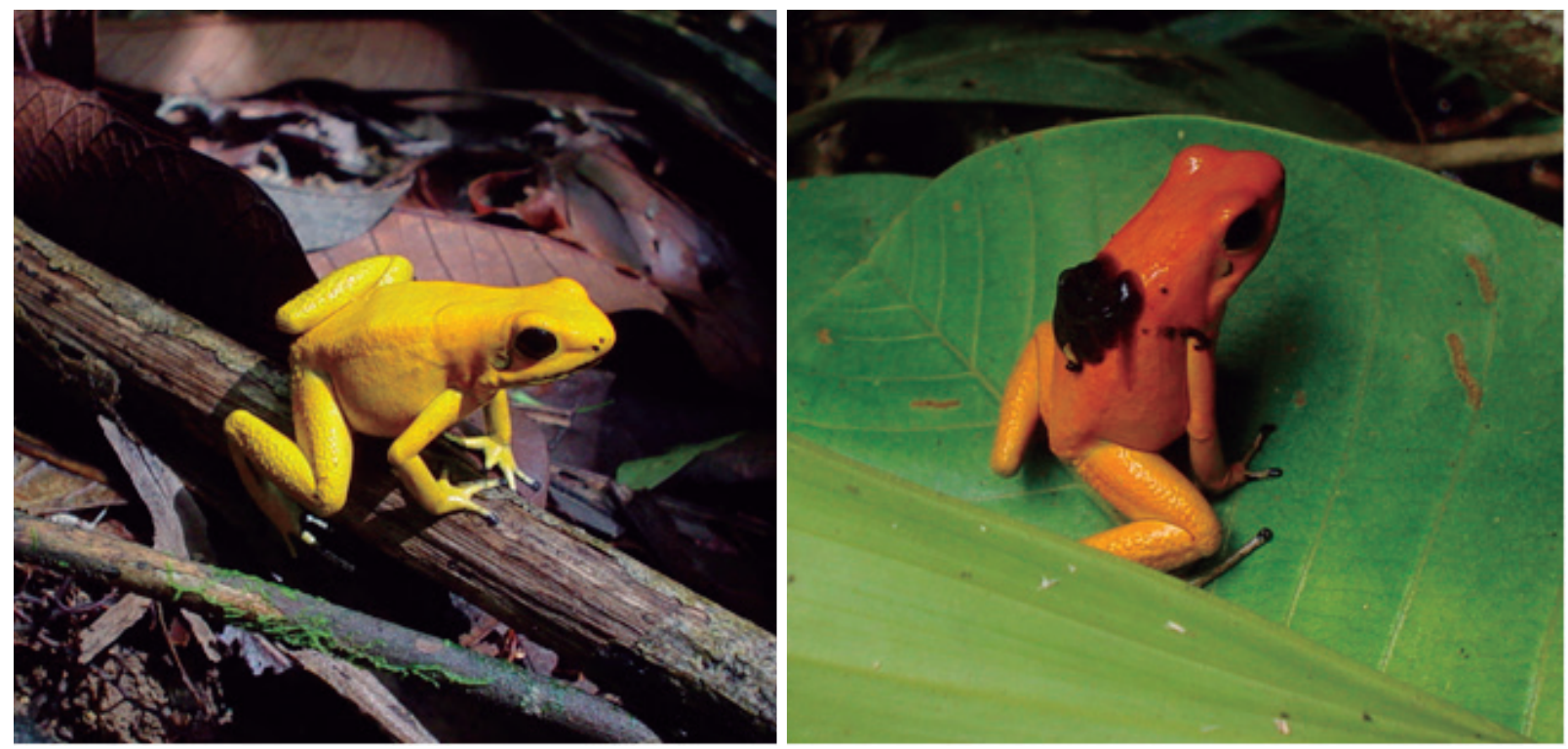

Figura 1. Individuos de Phyllobates terribilis en el resguardo indígena Joaquincito, Valle del Cauca, Colombia.

\section{Materiales y métodos}

Área de estudio. Esta investigación se realizó en Joaquincito, parte baja de la cuenca del río Naya, entre los departamentos del Valle del Cauca y Cauca $\left(3^{\circ} 17^{\prime} \mathrm{N}\right.$, y $\left.77^{\circ} 24^{\prime} \mathrm{O}\right)$ (Figura 2 y 3). Esta localidad se encuentra en la zona de vida de bosque pluvial tropical (bp-T) según el sistema Holdridge (Espinal y Montenegro, 1963) y se considera como un bosque primario intervenido y secundario (Ortega y Torres, 2013) (Figura 3). Presenta un rango altitudinal menor a 25 m s.n.m. y una temperatura promedio mayor a $24{ }^{\circ} \mathrm{C}$. El régimen pluviométrico es monomodal con precipitación media entre 6500 a $12000 \mathrm{~mm}$ (CVC, 2010). Para enero de 2015, aunque se esperaba un aumento de la probabilidad de ocurrencia del fenómeno de El Niño, se registraron temperaturas normales en el océano Pacífico a nivel general, según el Instituto de Hidrología, Meteorología y Estudios Ambientales (Ideam, www.ideam.gov.co). 


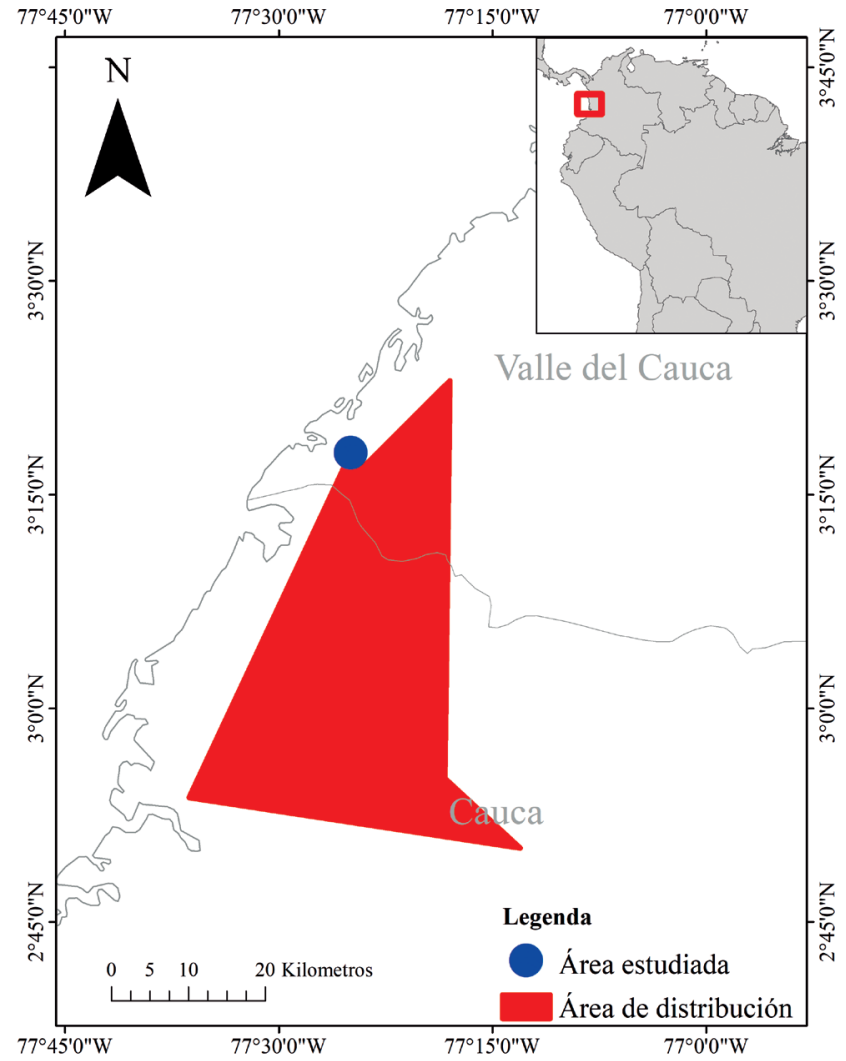

Figura 2. Mapa de la ubicación del departamento del Valle del Cauca, señalando la localidad de estudio correspondiente al resguardo indígena Joaquincito (punto azul).
Trabajo de campo. El muestreo fue realizado por 2 personas en una salida de campo entre el $19 \mathrm{y}$ 28 de enero de 2015. Se ubicaron 3 parcelas de 50 x $20 \mathrm{~m}\left(1000 \mathrm{~m}^{2}\right)$ separadas por aproximadamente $500 \mathrm{~m}$ una de otra, por lo cual se consideraron unidades independientes de muestreo. Se realizaron 4 revisiones por parcela dejando de muestrear cada parcela por 2 días consecutivos después de las primeras 2 sesiones. El muestreo iniciaba a las 07:00 horas hasta terminar toda el área correspondiente. El esfuerzo de muestreo fue de 72 horas/persona en un área total de $3000 \mathrm{~m}^{2}$. La búsqueda de individuos se realizó usando la técnica de Relevamiento por Encuentro Visual (REV) (Heyer et al., 1994), que consiste en buscar activamente sobre las raíces del suelo y hojarasca a los individuos presentes; estos se capturaron manualmente utilizando guantes de nitrilo para evitar posibles intoxicaciones.

A cada individuo observado y capturado se le midió su Longitud Rostro Cloaca (LRC) con ayuda de un calibrador digital 0,1 mm (Ubermann) para obtener una medida indirecta de la edad [adultos $(\geq 37,0 \mathrm{~mm})$, jóvenes $(\leq 36,9 \mathrm{~mm})$ ] (Myers et al., 1978; Lötters et al., 1997; Castro-Herrera, 2004). Todos los individuos capturados fueron marcados
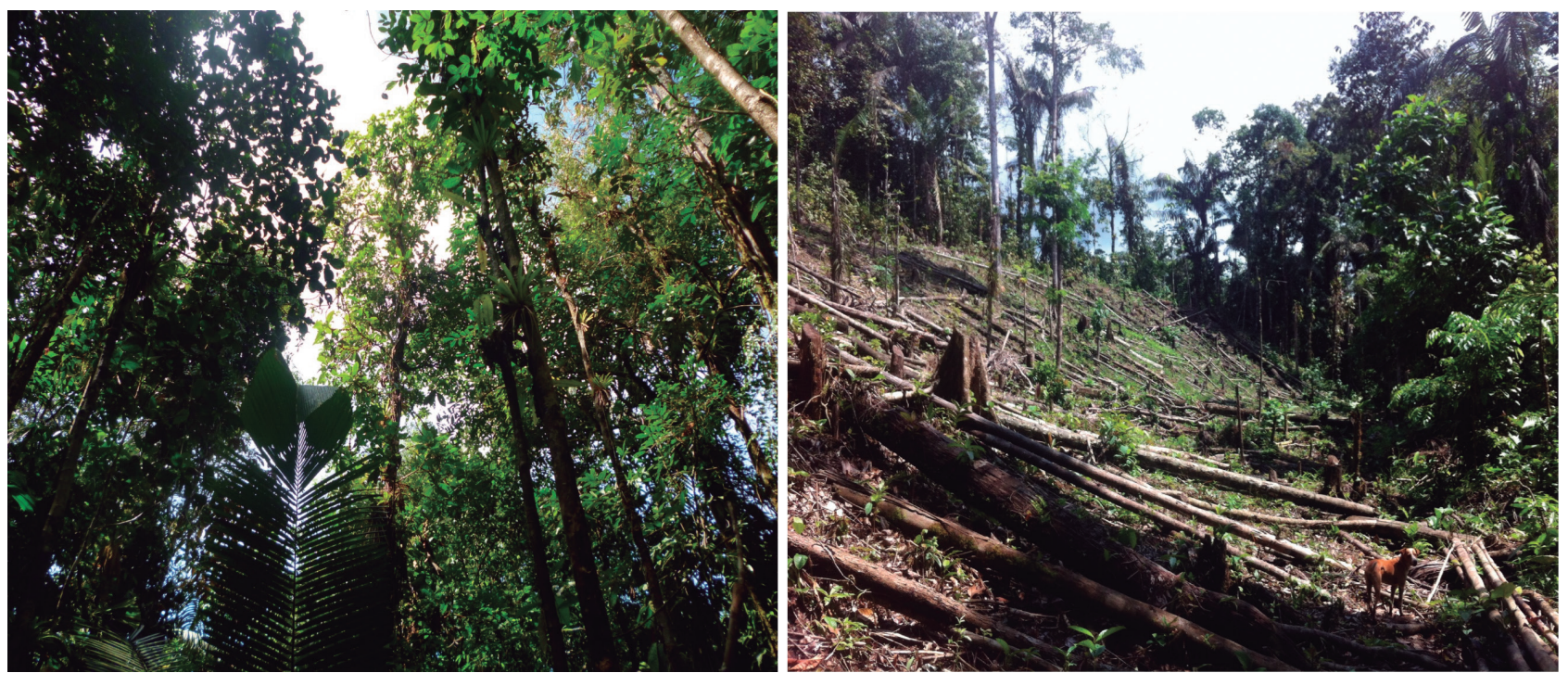

Figura 3. Área de estudio. Izquierda: interior de bosque. Derecha: borde de bosque con explotación de madera. 
empleando foto-identificación (Donnelly, 1994); para ello se utilizaron las manchas negras presentes en la parte ventral, las cuales no presentan un patrón regular, como marca para la identificación. Sin embargo, en los análisis solo se tuvieron en cuenta los individuos adultos debido a que en los jóvenes aún no era claro el patrón de coloración. Se tomaron fotografías del vientre de cada individuo después de su captura con una cámara digital Samsung Dv-150f, conservando el registro de sus marcas incluyendo todas las partes del cuerpo en la región ventral. Se tomaron dos fotografías por individuo en cada evento de captura y la recaptura se confirmó cuando el patrón de coloración ventral coincidió exactamente con la ubicación, forma y tamaño de los patrones de manchas previamente registradas para cada individuo (Figura 4). La precisión de la interpretación manual de los registros fotográficos ha sido demostrada en estudios anteriores (Kelly, 2001; Arzoumanian et al., 2005; Speed et al., 2007; Yánez-Muñoz, 2010; Loaiza-Piedrahíta et al., 2016). Este método se utilizó con el fin de minimizar los errores de identificación, ya que los análisis de CMR están fuertemente influenciados por la errónea identificación de individuos (Arnason y Mills, 1981).

Clasificación del hábitat. Cada parcela fue considerada una unidad independiente, se verificó y clasificó en cada una las condiciones de hábitat de acuerdo con la escala artificial propuesta por Manzanilla y La Marca (2004). Se identificaron las siguientes condiciones: a) ambiente prístino: sin perturbación aparente, con ausencia de establecimientos humanos, y sin contaminación sólida o líquida; b) ambiente ligeramente perturbado: con poca vegetación perturbada, densidad de población humana muy baja o inexistente y contaminación sólida o líquida baja o inexistente; c) ambiente modificado: hábitat natural fragmentado y presencia moderada de contaminantes sólidos y líquidos; y d) ambiente altamente degradado: con fragmentación de hábitat alta, y presencia de moderada a alta de contaminantes sólidos y líquidos. Adicionalmente, se realizó una descripción de cada parcela teniendo como referencia, tanto las observaciones de los investigadores como los datos proporcionados por la comunidad indígena que habita la zona.

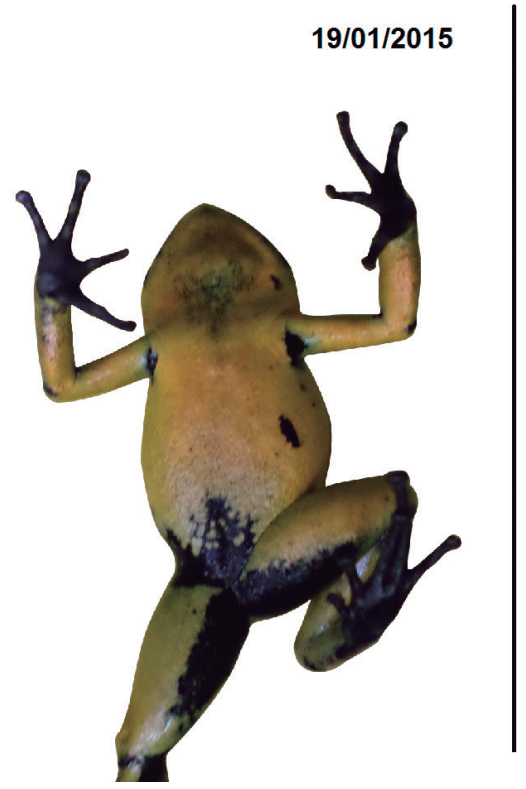

$28 / 01 / 2015$

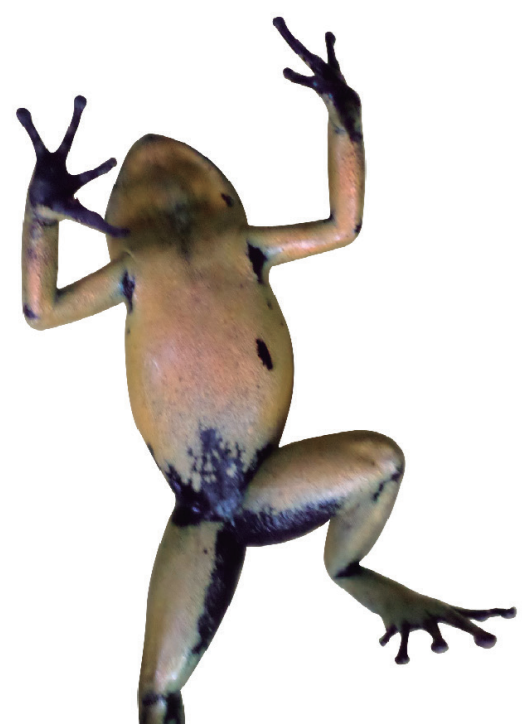

Figura 4. Ejemplo de un evento de recaptura de P.terribilis confirmado por la comparación de las fotografías tomadas en la fecha de la primera captura (izquierda) y segunda captura (derecha). 
Análisis de datos. La estimación del tamaño poblacional, la probabilidad de detección y de supervivencia se basaron en los datos obtenidos a partir del método de CMR, siendo una de las técnicas más precisas (Funk et al., 2003). La probabilidad de detección es importante para determinar si las posibles diferencias en las densidades poblacionales entre parcelas se deben a diferencias en la captura de los individuos o no. El método de CMR debe cumplir cuatro supuestos: 1) la muestra debe ser aleatoria, es decir que todos los individuos (marcados y no marcados) tienen igual probabilidad de ser capturados; 2) los individuos de la población deben tener homogeneidad de supervivencia; 3) las marcas deben ser permanentes (al menos durante el tiempo de la investigación) y debidamente registradas y; 4) los individuos capturados, deben ser liberados inmediatamente, una vez finalizado el muestreo (Amstrup et al., 2005).

Se construyeron historias de captura para cada uno de los individuos registrados durante el muestreo con base en los datos de CMR. Posteriormente, las historias de captura se analizaron a través del modelo de Jolly-Seber (White y Burnham, 1999) utilizando la parametrización implementada en la formulación POPAN (Arnason y Schwarz, 1995). Este modelo incluye los parámetros de tamaño poblacional $(\mathrm{N})$, probabilidad de entrada a la población (pent), la probabilidad de supervivencia $(\varphi)$ y la probabilidad de detección $(p)$. Para todos los parámetros se consideró un modelo nulo, que representa la no variación en los parámetros. Además, se corrieron todas las combinaciones posibles de los modelos, es decir, incluyendo cada parámetro como constante (.) y variable (pr). El efecto de la parcela sobre el tamaño poblacional fue incluido en el análisis cuando se considera el $\mathrm{N}$ como variable $(p r)$.

Para la selección de los modelos y la inferencia estadística acerca de la influencia de los factores sobre los parámetros del modelo se utilizó el Criterio de Información de Akaike corregido para muestras pequeñas - AICc (Burnham, 2004). Se calcularon las diferencias en AIC ( $\triangle \mathrm{AICc}$ ) y el peso del AICc y se utilizaron para evaluar el soporte relativo que recibieron los modelos (Burnham y Anderson, 2002). Los análisis fueron realizados en el programa MARK (White y Burnham, 1999) usando el paquete RMark en el lenguaje computacional R (Core Team, 2017). Finalmente, la densidad poblacional se estimó dividiendo el tamaño poblacional entre el área total de las parcelas.

\section{Resultados}

Parámetros poblacionales. Se capturaron un total de 36 individuos (26 adultos y 10 juveniles) en la zona de estudio durante el periodo de muestreo. La Longitud Rostro-Cloaca (LRC) promedio fue de 38,7 $\mathrm{mm} \pm 3,4$ (IC $95 \%$ : 37,4 a 40,0 mm); la talla mínima fue de $29,8 \mathrm{~mm}$ y la máxima de $45,8 \mathrm{~mm}$. De estos, 15 fueron capturados en una sola ocasión $(57,7 \%), 7$ en 2 ocasiones (26,9\%), 2 en 3 ocasiones $(7,6 \%)$, y 2 individuos en 4 (7,6\%). De acuerdo con los resultados, el modelo que presentó el AIC más bajo no incluyó ningún tipo de variación para los 4 parámetros (Modelo 1, Tabla 1). Adicionalmente, como los modelos restantes se separaron de este con valores superiores a 2 unidades de AIC, los resultados indican que el modelo 1 se ajustó mejor a los datos, resultando ser el más parsimonioso.

El tamaño de la población fue constante en las 3 parcelas. Este resultado es soportado por las estimaciones poblacionales del modelo más parsimonioso (el valor de AIC menor) que incluyó un posible efecto de las parcelas (par) sobre el tamaño poblacional (Modelo 3, Tabla 1). De acuerdo con las estimaciones del tamaño poblacional derivadas de dicho modelo, el traslape de los intervalos de confianza fue considerable (Tabla 2), lo cual indica que no hubo una variación que apoye un efecto de las parcelas sobre el tamaño poblacional. El tamaño poblacional total fue de $32,5 \pm 4,3$ ind. con un intervalo de confianza de 95 $\%$ de $27,9-47,5$ ind. (promedio \pm error estándar), mientras que la densidad estimada fue de 0,011 ind. $/ \mathrm{m}^{2}$ con un intervalo de confianza de $95 \%$. 
Tabla 1. Modelos de estimación del tamaño poblacional de $P$. terribilis en Joaquincito, Buenaventura, Colombia. Modelo de Jolly-Seber (parametrización de POPAN), donde: $\varphi=$ Probabilidad de supervivencia aparente, $p=$ Probabilidad de detección, pent=Probabilidad de entrada de un individuo entre la ocasión $i$ y $i+1$ y $N=$ Tamaño poblacional, $()=$. constante,$(\mathrm{t})=$ variable,$(\mathrm{pr})=$ parcela.

\begin{tabular}{lcccc}
\hline \multicolumn{1}{c}{ Modelo } & $\begin{array}{c}\text { Número de } \\
\text { parámetros }\end{array}$ & AIC & $\begin{array}{c}\text { Delta } \\
\text { AIC }\end{array}$ & $\begin{array}{c}\text { Peso } \\
\text { AIC }\end{array}$ \\
\hline $1 \varphi() p.(.) \operatorname{pent}() N.()$. & 4 & 108,415 & 0 & 0,769 \\
\hline $2 \varphi(t) p(.) \operatorname{pent}() N.()$. & 6 & 112,402 & 3,986 & 0,104 \\
\hline$\varphi() p.(.) \operatorname{pent}() N.(p r)$ & 6 & 113,316 & 4,9 & 0,066 \\
\hline $4 \varphi() p.(t) \operatorname{pent}() N.()$. & 7 & 113,87 & 5,454 & 0,05 \\
\hline $5 \varphi(t) p(.) \operatorname{pent}() N.(p r)$ & 8 & 117,985 & 9,569 & 0,006 \\
\hline $6 \varphi() p.(t) \operatorname{pent}() N.(p r)$ & 9 & 119,756 & 11,34 & 0,002 \\
\hline
\end{tabular}

Tabla 2. Tamaño poblacional de $P$. terribilis en la localidad de Joaquincito, Buenaventura, Colombia. Tamaño de población estimado con base en el modelo más parsimonioso que incluyó el efecto de las parcelas sobre el tamaño poblacional (modelo 3, tabla 1): $\varphi() p.($. pent(.) $N$ (par). Intervalo de confianza de $95 \%$.

\begin{tabular}{cccccc}
\hline Parcela & N & $\begin{array}{c}\text { Error } \\
\text { estándar }\end{array}$ & Mínimo & Máximo & $\begin{array}{c}\text { Densidad } \\
\text { (ind/m2 }\end{array}$ \\
\hline 1 & 9,25 & 1,73 & 8,16 & 14,56 & 0,009 \\
\hline 2 & 6,8 & 1,45 & 6,07 & 17,51 & 0,007 \\
\hline 3 & 14,1 & 2,25 & 12,4 & 23,61 & 0,014 \\
Total & $\mathbf{3 2 , 5}$ & $\mathbf{4 , 3}$ & $\mathbf{2 7 , 9}$ & $\mathbf{4 7 , 5}$ & $\mathbf{0 , 0 1 1}$ \\
\hline
\end{tabular}

Con base en el modelo más parsimonioso, las probabilidades de supervivencia y de detección fueron constantes en las 3 parcelas; correspondieron a un estimado de supervivencia aparente igual a 0,74 \pm 0,08 (IC $95 \%$ : 0,54 - 0,88) y a un estimado de la probabilidad de detección de 0,56 $\pm 0,13$ (IC $95 \%=0,30-0,78$ ). Esto puede indicar que las similitudes en las densidades y los tamaños poblacionales entre parcelas son reales, $y$ no producto de diferencias en la probabilidad de detección y supervivencia de los individuos, como consecuencia de diferencias en las características ambientes de las parcelas $\mathrm{u}$ otros factores.

Clasificación del hábitat. La parcela 1 fue categorizada con la condición c (ambiente modificado, a pesar de observarse baja contaminación sólida), en donde predominó el dosel abierto y 
estuvo junto a un camino muy transitado al lado de una huerta comunitaria, por lo cual era un ambiente fragmentado y el grado de perturbación era alto respecto a las parcelas 2 y 3 . Estas últimas se ajustaron a la condición b (ambiente ligeramente perturbado) con predominio de dosel cerrado y con profundidad de la hojarasca de $15 \mathrm{~cm}$ en promedio. En las 3 parcelas se reportó alta presencia de fuentes de agua y lugares potenciales de refugio para $P$. terribilis. Según las encuestas realizadas a los integrantes de la comunidad indígena, el tránsito por caminos cercanos a la parcela 2 era ocasional debido a que, a pocos metros, se ubicaba un sector de extracción maderera; mientras que en la parcela 3 el transito era escaso.

\section{Discusión}

En este estudio, los tamaños poblacionales no presentaron diferencias cuando se evaluaron entre parcelas, a pesar de las diferencias en el grado de modificación del hábitat que presentó una de estas. Considerando que tampoco se observaron diferencias significativas entre las probabilidades de supervivencia y de detección entre parcelas, es posible que la modificación del hábitat observada no sea un factor determinante en la densidad y tamaño poblacional de $P$. terribilis, pues a pesar de la perturbación, las tres parcelas presentaron fuentes de agua y lugares potenciales de refugio. Estos resultados también pueden indicar que el área de estudio aún conserva características de composición, estructura y función que permiten la subsistencia de esta especie con distribución restringida a áreas conservadas.

En anuros, la presencia de especies en hábitats particulares está determinada por la disposición de recursos alimenticios, refugio, sitios de termorregulación, reproducción, entre otros, que son necesarios para cumplir con sus requerimientos fisiológicos (Zug et al., 2001; UrbinaCardona et al., 2006). La disponibilidad de esos recursos pueden determinar la respuesta de las especies a la fragmentación del hábitat (Malcolm, 1997; Primak et al., 2001) o a la degradación de bosques primarios a secundarios (Pröhl y Berke, 2001). Además de la disponibilidad de recursos, hay otras condiciones ambientales que pueden permitir el establecimiento de especies en ecosistemas perturbados. Urbina y Londoño-M (2003), observaron que el $92 \%$ de las especies de anfibios y reptiles encontradas en bosque primario también estaban en bosque secundario con características microclimáticas similares. En la región Pacífica, Vargas-S y Bolaños-L (1999), hallaron diferencias en la estructura, composición y riqueza de anfibios entre áreas boscosas maduras y secundarias con áreas de cultivos con vegetación herbácea y arbustiva. Ellos atribuyeron esas diferencias a la variación en las condiciones de humedad entre las áreas analizadas, puesto que hábitats con poca estructura vegetal pueden generar estrés fisiológico para muchas especies. Por lo tanto, la ausencia de diferencias significativas en los parámetros estimados entre parcelas con diferente grado de intervención, puede deberse a que los recursos disponibles y las condiciones microclimáticas, que aún persisten, permiten el establecimiento de poblaciones viables de $P$. terribilis. Sin embargo, es necesario evaluar la dinámica de estos parámetros a largo plazo donde se incluya la variación anual de las condiciones ambientales, así como los efectos de fenómenos climáticos como El Niño y La Niña, que pueden modificar la disposición de recursos y el microclima especialmente en los bordes de las áreas fragmentadas.

No obstante, la literatura científica cuenta con poca información referente a las características del hábitat de $P$. terribilis. En el departamento del Cauca, se reporta su presencia en bosques poco perturbados y bosques nativos casi intactos y se conoce que evita los bosques de sucesión secundaria y áreas cultivadas (Myers et al., 1978; Castro-Herrera, 2004). Sin embargo, el presente estudio fue realizado en un bosque clasificado como primario intervenido y secundario (Ortega y Torres, 2013). Adicionalmente, esta especie se encontró en parcelas que no solo tenían algún grado de perturbación (dosel abierto), si no que estaban junto a zonas de perturbación constante 
(caminos y huertas comunitarias). Según Hunter y Gibbs (2007), dependiendo de las características de distintas poblaciones de una misma especie, estas pueden responder de manera diferente a los disturbios del hábitat. Además, Tocher et al. (1997) afirman que, a partir de un trabajo de diez años en la Amazonia central brasilera, muchas especies no se encuentran verdaderamente aisladas en bosques fragmentados cuando la matriz presenta condiciones adecuadas para la migración de anfibios entre fragmentos. Estos resultados sugieren que, aunque anteriormente $P$. terribilis solamente se haya observado en áreas conservadas, es posible que algunas poblaciones presenten niveles de tolerancia a ambientes con cierto grado de modificación, como la estudiada en la localidad de Juaquincito. Por lo tanto, es necesario medir el efecto del estado sucesional del bosque sobre las poblaciones de P. terribilis, así como la respuesta poblacional de esta especie a diferentes grados de fragmentación del hábitat. Estos resultados sirven como punto de referencia para futuras investigaciones enfocadas a evaluar el estado poblacional de $P$. terribilis en la localidad de Juaquincito, resaltando la importancia de incluir la variación temporal.

La probabilidad de detección registrada $(p=56 \%)$, además de estar fuertemente influenciada por la duración del estudio, el tamaño corporal y los hábitos territoriales, está afectada por la coloración aposemática que pueden hacer de $P$. terribilis, una especie con alta detectabilidad en comparación con otras especies de la misma familia. En especies de dendrobatidae con LRC inferiores a $20 \mathrm{~mm}$, como en Andinobates opisthomelas (Boulenger, 1899) y Andinobates minutus (Shreve, 1935), se han encontrado probabilidades de detección máximas de 1,7 y 28 \% respectivamente (Lozano-Osorio, 2015; Loaiza-Piedrahíta et al., 2016); mientras que en especies con un promedio de LRC menor a 26 $\mathrm{mm}$, como Colostethus aff. fraterdanieli (Silverstone, 1971) y Dendrobates truncatus (Cope, 1861), se observó una probabilidad de detección de 42,6 y 58,8 \% (Molina-Zuluaga et al., 2014). Considerando que $P$. terribilis es una de las especies de mayor tamaño entre los dendrobátidos, puesto que presenta una LRC 41,05 \pm 0,11 $\mathrm{mm}$ (Myers et al., 1978), es posible que exista un efecto del tamaño corporal sobre la probabilidad de detección en especies de esta familia, y que esta probabilidad de detección aumente con el tiempo como fue registrado para $A$. minutus en donde se observó un valor de 0,13 (IC $95 \%$ : 0,10-0,16) en el primer evento de muestro y 0,28 (IC $95 \%$ : 0,24-0,33) después de 6 meses (Lozano-Osorio, 2015). En consecuencia, se espera una alta probabilidad de detección en trabajos con muestreos a mediano y largo plazo sobre $P$. terribilis, por lo que esta es una especie ideal para evaluar el efecto a largo plazo de las características del hábitat sobre la probabilidad de supervivencia y el tamaño poblacional en dendrobátidos.

En este estudio se obtuvo un valor estimado de probabilidad de supervivencia aparente de 0,74 $\pm 0,08$ que está relacionado con la duración del muestreo, puesto que en trabajos más prolongados en el tiempo, es más probable observar eventos de mortalidad por depredación, enfermedad o por vejes de los individuos. A pesar de que la probabilidad calculada pueda estar influenciada por el corto tiempo de muestreo, este estudio ofrece algunas perspectivas sobre la probabilidad de supervivencia de $P$. terribilis, considerando la importancia de esta especie y la poca información sobre ella que está disponible. Se espera que esta probabilidad no varíe drásticamente a mediano plazo si se considera el tamaño corporal y el bajo aporte de la depredación sobre el número de muertes en esta especie, pues las secreciones de la piel de $P$. terribilis presentan batracotoxinas, homobatracotoxinas y alcaloides esteroideos que son muy fuertes y tóxicos para sus depredadores (Myers et al., 1978; Pröhl, 2005). En poblaciones de especies de la misma familia ya se ha observado un efecto del tamaño corporal sobre la supervivencia a nivel intrapoblacional, en donde los individuos más grandes tienen mayores probabilidades de sobrevivir (Loaiza-Piedrahíta et al., 2016). Para la población de $P$. terribilis objeto de estudio, se obtuvieron valores de LRC entre 37,4 y 40,0 mm, la cual corresponde a individuos adultos (Myers et al., 1978; Lötters et al., 1997; Castro-Herrrera, 
2004). Esta clase etaria es la que presentan la menor mortalidad en anfibios (Hutchinson, 1981). Sin embargo, es de esperarse que este valor disminuya gradualmente con el tiempo debido a que aumenta la posibilidad de que los individuos mueran por eventos estocásticos, depredación o enfermedad. La disminución de la probabilidad de supervivencia, con relación al tiempo, ha sido observada en Andinobates minutus de una localidad insular del Pacífico colombiano, en donde esta probabilidad varió de 0,97 (IC $95 \%$ : 0,94-0,98) a 0,83 (IC $95 \%: 0,76-0,88$ ) en 6 meses de estudio (Lozano-Osorio, 2015). Además de estos factores, es importante considerar posibles comportamientos territoriales y de fidelidad de hábitat en $P$. terribilis que puedan influenciar su probabilidad de supervivencia, así como ya se ha propuesto para otras especies de la misma familia (Molina-Zuluaga et al., 2014; Lozano-Osorio, 2015).

\section{Conclusiones y recomendaciones}

La densidad y el tamaño poblacional calculados para la población de $P$. terribilis en el resguardo indígena Juaquincito, Valle del Cauca, Colombia, pueden tener mayor relación con la disponibilidad de recursos o con condiciones microclimáticas específicas para esta especie, que con la modificación del hábitat cuando esta es moderada.

Las probabilidades de detección y supervivencia de esta población pueden estar asociadas con la corta duración del muestreo; por lo tanto, es necesario describir estos parámetros a largo plazo donde sea incluida la temporalidad y la variación climática anual. Además de la temporalidad, estos parámetros también pueden ser influenciados por la baja depredación, sus hábitos territoriales, su tamaño corporal y su coloración aposemática. Por ende, es necesario realizar estudios ecológicos, etológicos y de historia natural, así como medir el efecto del estado sucesional del bosque y la fragmentación sobre las poblaciones de $P$. terribilis.

Este trabajo constituye el primer análisis demográfico de $P$. terribilis en Colombia, y sirve como punto de referencia para la comprensión de su dinámica temporal en futuros estudios; además de aportar información necesaria para el desarrollo de estrategias de conservación in situ.

Se propone que, para estudios posteriores con esta especie, en los que se incluya CMR, se considere el método de foto-identificación, pues resultó efectivo en este estudio; de esta manera se estaría evitando el empleo de métodos que pueden resultar invasivos, como la remoción de falanges. Finalmente, se recomienda seguir un monitoreo en la zona de estudio y lugares aledaños para determinar el estado y la dinámica de las poblaciones a mediano y largo plazo. Esto facilitaría la elección de las áreas prioritarias para la conservación en el Sistema Regional de Áreas Protegidas (SIRAP) del municipio, mejorando así las medidas para la conservación de esta especie endémica de Colombia.

\section{Agradecimientos}

Este trabajo contó con el apoyo financiero de de la Asociación Colombiana de Herpetología (ACH) (por medio de la iniciativa Botas al campo) y la Fundación Zoológica de Cali. Agradecemos a la comunidad indígena de Joaquincito por permitirnos el ingreso a la zona durante el periodo de estudio, a Jorge Kelvin Torres por su colaboración durante la recolección de datos; a Alejandro Valencia Zuleta por la elaboración del mapa; a Oscar Murillo por su asesoría científica y a Mario Fernando Garcés Restrepo por la revisión crítica del trabajo de investigación.

\section{Referencias}

Amstrup, S., McDonald, T. y Manly, B. (2005). Handbook of capture-recapture analysis. New Jersey: Princeton University Press. 313 pp.

Arnason, N. A., y Schwarz, C. J. (1995). POPAN-4: enhancements to a system for the analysis of mark-recapture data from open populations. Journal of Applied Statistics, 22, 785-800. 
Arnason, A. N. y Mills, K. H. (1981). Bias and loss of precision due to tag loss in Jolly-Seber estimates for mark-recapture experiments. Canadian Journal of Fisheries and Aquatic Science, 38, 1077-1095.

Arzoumanian, Z., Holmberg, J. y Norman, B. (2005). An astronomical pattern-matching algorithm for computer-aided identification of whale sharks Rhincodon typus. Journal of Applied Ecology, 42, 999-1011.

Barlett, R. D. (2003). Poison dart frogs. New York: Barron's Educational Series. 12 pp.

Berman, B. (2004). Complementary and alternative medicine: is it just a case of more tools for the medical bag? The Clinical Journal of Pain, 20, 1-2.

Blaustein, A. R., Han, B., Relyea, R., Johnson, P. T., Buck, J. C., Gervasi, S. S. y Kats, L. B. (2011). The complexity of amphibian population declines: understanding the role of cofactors in driving amphibian losses. Annals of the New York Academic of Science, 1223(1), 108-119.

Boulenger, G. A. (1899). Descriptions of new batrachians in the collection of the British Museum (Natural History). Journal of Natural History Series, 7(3), 273-277.

Brown, J. L, Twomey, E., Amézquita, A., De Souza, M., Caldwell, J., Lötters, S., May, R., MeloSampaio, P., Mejía-Vargas, D., Perez-Peña, P., Pepper, M., Poelman, E. H., Sanchez-Rodriguez, M. y Summers, K. (2011). A taxonomic revision of the Neotropical poison forg genus Ranitomeya (Amphibia: Dendrobatidae). Zootaxa, 3083, 1-120.

Burnham, K. P. (2004). Multimodel Inference: Understanding AIC and BIC in Model selection. Sociological Methods \& Research, 33, 261-304.

Burnham, K. P. y Anderson, D. R. (2002). Model selection and inference: a practical informationtheoretic approach. Segunda edición. New York: Springer. 448 pp.

Castro-Herrera, F. (2004).Rana venenosa dorada: Phyllobates terribilis. En Rueda-Almonacid, J. V., Lynch, J. D y Amézquita, A. (Eds.) Libro rojo de los anfibios de Colombia. Pp.178-181. Bogotá:
Conservación Internacional Colombiana, Universidad Nacional, Instituto de Ciencias Naturales y Ministerio del Medio Ambiente.

CVC. (2010). Análisis preliminar de la representatividad ecosistémica, a través de la recopilación, clasificación, $y$ ajuste de información primaria y secundaria con rectificaciones de campo del mapa de ecosistemas de Colombia para la jurisdicción del Valle del Cauca. (Informe técnico). 243 pp.

Donnelly, M. A. (1994). Demographic effects of reproductive resource supplementation in a territorial frog Dendrobates pumilio. Ecological Monographs, 59(3), 207-221.

Espinal, L. S. y Montenegro, E. (1963). Formaciones vegetales de Colombia y mapa ecológico: memoria explicativa. Bogotá: Instituto Geográfico Agustín Codazzi, Subdirección Agrológica. 201 pp.

Funk, W. C, Almeida, D., Nogales, F., y Bustamante, M. R. (2003). Monitoring population trends of Eleutherodactylus frogs. Journal of Herpetology, 37(2), 245-256.

Grant, T., Frost, D. R., Caldwell, J. P., Gagliardo, R., Haddad, C. F. B., Kok, P. J. R., Means, D. B., Noonan, B. P., Schargel, W. E. y Wheeler, W. C. (2006). Phylogenetic systematics of dart-poison frogs y their relatives (Amphibia: Athesphatanura: Dendrobatidae). Bulletin of the American Museum of Natural History, 299, 1-262.

Heyer, W. R., Donnelly, M. A., McDiarmid, R. W., Hayek, L. C. y Foster, M. S. (1994). Measuring y Monitoring Biological Diversity: Standard Methods for Amphibians. Washington: Smithsonian Institution Press. 364 pp.

Hunter, J. R. y Gibbs, J. P. (2007). Fundamentals of Conservation Biology. Tercera edición. Malden, Massachusetts: Blackwell publishing. 497 pp.

Hutchinson, G. E. (1981). Introducción a la ecología poblacional. Barcelona: Editorial Blume. 250 pp.

IUCN SSC Amphibian Specialist Group. (2017). Phyllobates terribilis. The IUCN Red List of Threatened Species 2017: e.T55264A85887889. Recuperado el 30 de marzo 
de 2018 de http://dx.doi.org/10.2305/IUCN. UK.2017-3.RLTS.T55264A85887889.en.

Kelly, M. J. (2001). Computer-aided photograph matching in studies using individual identification: an example from Serengeti cheetahs. Journal of Mammalogy, 82, 440-449.

Lips, K. R., Reaser, J. K., Young, B. E. e Ibáñez R. (2001). Amphibian Monitoring in Latin America: A Protocol Manual. Florida: Society for the study of amphibians and reptiles. $115 \mathrm{pp}$.

Loaiza-Piedrahíta, J. D., Bock, B. C. y Páez, V. P. (2016). Demography of the Andean Dart Frog (Andinobates opisthomelas, Dendrobatidae) in Eastern Antioquia, Colombia. South American Journal of Herpetology, 11(2), 81-88.

Lötters, S., Castro-Herrera, F., Köhler, J. y Richter, R. (1997). Notes on the distribution y color variation of poison frogs of the genus Phyllobates from western Colombia (Anura: Dendrobatidae). Revue Francais Aquariologie, 24, 55-58.

Lozano-Osorio, R. (2015). Parámetros poblacionales de Andinobates minutus (Shreve 1935) (anura: Dendrobatidae) en isla palma, PNN Urámba Bahía Málaga, Valle del Cauca. (Trabajo de grado). Cali: Universidad del Valle, Facultad de Ciencias Naturales, Departamento de Biología. 9 pp.

Malcolm, J. R. (1997). Biomass and diversity of small mammals in Amazonas forest fragments: En Laurence, W.F., Bierregard, R.O. (Eds.) Tropical forest remnants: Ecology, Management, and Conservation of Fragmented Communities. Pp. 207221. Chicago: University of Chicago Press.

Marín, O. H. y Gómez-Hoyos, D. A. (2011). Estado actual de Ranitomeya bombetes (Anura: Dendrobatidae). Plan de manejo y conservación para las poblaciones de Ranitomeya bombetes (Myers y Daly, 1980) en Quindío Colombia. Saarbrücken: Editorial académica española. 108 pp.

Manzanilla, J. y La Marca, E. (2004). Population status of the Rancho Grande Harlequin frog (Atelopus cruciger Lichtenstein and martens 1856), a proposed critically endangered species from the Venezuelan coastal range. Memoria de la Fundación La Salle de Ciencias Naturales, 62, 5-29.

Márquez, R., Corredor, G., Galvis, C., Góez, D. y Amézquita, A. (2012). Range extension of the critically endangered true poison-dart frog, Phyllobates terribilis (Anura: Dendrobatidae), in western Colombia. Acta Herpetológica, 7(2), 341345.

Molina-Zuluaga, C., Restrepo, A., Flechas, S. V. y Daza, J. D. (2014). Short-term population dynamics of three frog species in the Northern Andes, Colombia. South American Journal of Herpetology, 9(3), 200-206.

Myers, C. W., Daly, J. W. y Malkin, B. A. (1978). Dangerously toxic new frog (Phyllobates) used by Emberá Indians of western Colombia, with discussion of blowgun fabrication y dart poisoning. Bulletin of the American Museum of Natural History, 161, 307-366.

Ortega, J. F. y Torres, A. M. (2013). Productos forestales no maderables en dos comunidades socioculturales en el Pacífico vallecaucano. En Sanabria, O. L. (Ed). Valoración del conocimiento, uso, manejo y prácticas de conservación de la diversidad de recursos forestales no maderables en diferentes ambientes socioculturales de la región del Pacífico Colombiano. Bogotá: Asociación Colombiana de Herbarios.

Pennisi, E. (1992). Pharming frogs. Science News, 142(3), 40-42.

Pounds, J. A., Bustamante, M. R., Coloma, L. A., Consuegra, J. A., Fogden, M. P. L., Foster, P. N., La Marca, E., Masters, K. L., Merino-Viteri, A., Puschendorf, R., Ron, S. R., Sanchez-Azofeifa, G. A., Still, C. J. y Young, B. E. (2006). Widespread amphibian extinctions from epidemic disease driven by global warming. Nature, 439, 161-167.

Primack, R., Rozzi, R., Massardo, F. y Feinsinger, P. (2001). Destrucción y degradación del Hábitat. En Primack, R., Rozzi, R., Feinsinger, P., Dirzo, R., Massardo, F. (Eds.) Fundamentos de conservación biológica. Perspectivas latinoamericanas. Pp. 183- 
224. Ciudad de México: Fondo de Cultura Económica.

Pröhl,H.(2005). Territorial Behavior in Dendrobatid Frogs. Journal of Herpetology, 39, 354-365.

Pröhl, H. y Berke, O. (2001). Spatial Distributions of Male and Female Strawberry Poison Frogs and Their Relation to Female Reproductive Resources. Oecologia, 129, 534-542.

Core Team . (2017). R: A language and environment for statistical computing. Vienna, Austria: R Foundation for Statistical Computing.

Ríos, S. C., Puerta, P. y Sierra, E. (2011). Densidad poblacional de Ranitomeya opisthomelas y su relación con variables ambientales y de hábitat en cuatro bosques de la cordillera Central de Colombia. Boletin Científico Museo de Historia Natural de la Universidad de Caldas, 15(2), 121-129.

Ruiz-Carranza, P. M., Ardila-Robayo, M. C. y Lynch, J. D. (1996). Lista actualizada de la fauna de Amphibia de Colombia. Revista de la Academia Colombiana de Ciencias Exactas, Fisicas y Naturales 20(77), 365-415.

Speed, C. W., Meekan, M. G. y Bradshaw, C. J. (2007). Spot the match-wildlife photoidentification using information theory. Frontiers in Zoology, 4(2), 1-11.

Santos, J. C., Coloma, L. A. y Cannatella, D. C. (2003). Multiple, recurring origins of aposematism and diet specialization in poison frogs. Proceeding of the National Academic of Sciences of the United States of America 100(22), 12792-12797.

Saporito, R. A., Garraffo, H. M., Donnelly, M. A., Edwards, A. L., Longino, J. T. y Daly, J. W. (2004). Formicine ants: An arthropod source for the pumiliotoxin alkaloids of dendrobatid poison frogs. Proceeding of the National Academic of Sciences of the United States of America, 101(21), 8045-8050.

Silverstone, P. A. (1971). Status of certain frogs of the genus Colostethus, with descriptions of new species. Contributions in Science. Natural History Museum of Los Angeles County, 215, 1-8.
Tocher, M. D., Gascon, C. y Zimmerman, B. L. (1997). Fragmentation effects on a central Amazonian frog community: a 10-year study. En: Laurance, W. F. y. Bierregaard, R. O. (Eds.). Tropical forest remnants: ecology, management, and conservation of fragmented communities. Pp. 124137. Chicago: University of Chicago Press.

Urbina-C., J. N. y Londoño-M, M. C. (2003). Distribución de la comunidad de herpetofauna asociada a cuatro áreas con diferente grado de perturbación en la isla Gorgona, Pacífico colombiano. Revista de la Academia Colombiana de Ciencias Exactas, Físicas y Naturales, 27(102), 105-113.

Urbina-Cardona, J. N., Olivares-Pérez, M. y Reynoso, V. H. (2006). Herpetofauna diversity and microenvironment correlates across the pasture-edge-interior gradient in tropical rainforest fragments in the region of Los Tuxtlas, Veracruz. Biological Conservation, 132, 61-75.

Vargas, F. y Bolaños, M. E. (1999). Anfibios y reptiles presentes en hábitats perturbados de selva lluviosa tropical en el bajo Anchicayá, Pacífico colombiano. Revista de la Academia Colombiana de Ciencias Exactas, Físicas y Naturales, 23, 499-511.

Vitt, L. J. y Caldwell, J. (2009). Herpetology: an introductory biology of amphibians and reptiles. San Diego: Academic Press. 776 pp.

White, G. C. y Burnham, K. P. (1999). Program MARK: survival estimation from populations of marked animals. Bird Study, 46, 120-138.

Yánez-Muñoz, M. H., Meza-Ramos, P., Altamirano, M. y Castro, C. (2010). Estado poblacional de una de rana nodriza (Anura: Dendrobatidae: Hyloxalus delatorreae), críticamente amenazada, en los Andes norte de Ecuador. Boletín Técnico Serie Zoologica, 6, 38-64.

Young, B. E., Lips, K. R., Reaser, J. K., Ibáñez, R., Salas, A. W., Cedeño, J. R., Coloma, L. A., Ron S., La Marca, E., Meyer, J. R., Muñoz, A., Bolaños, F., Chaves, G. y Romo, D. (2001). Population declines y priorities for amphibian conservation 
in Latin America. Conservation Biology, 15 (5), 1213-1223.

Young, B. E., Stuart, S. N., Chanson, J. S., Cox, N. A. y Boucher, T. M. (2004). Joyas que están desapareciendo: el estado de los anfibios en el Nuevo
Mundo. Virginia: NatureServe. 53 pp.

Zug, G. R., Vitt, L. J. y Caldwell, J. P. (2001). Herpetology: An Introductory Biology of Amphibians and Reptiles. Segunda edición. California: Academic press.
Stefhania Alzate-Lozano

Universidad del Valle,

Departamento de Biología

Cali, Colombia

stefhania.alzate@correounivalle.edu.co

\section{Rodrigo Lozano-Osorio}

Universidad Federal de Pará, Instituto de Ciencias Biológicas

Pará, Brasil

lozano.osorio.r@gmail.com

\section{Carlos Galvis}

Fundación Zoológica de Cali

Cali, Colombia

carlosgalvis@zoologicodecali.com.co
Parámetros poblacionales de la rana dorada, Phyllobates terribilis (Myers et al., 1978) (Dendrobatidae), en una localidad de Buenaventura, Valle del Cauca, Colombia

Citación del artículo: Alzate-Lozano, S., LozanoOsorio, R. y Galvis, C. (2018). Parámetros poblacionales de la rana dorada, Phyllobates terribilis (Myers et al., 1978) (Dendrobatidae), en una localidad de Buenaventura, Valle del Cauca, Colombia. Biota Colombiana, 19(1), 133-146. DOI: 10.21068/c2018.v19n01a09.

Recibido: 6 de julio de 2017

Aprobado: 20 de abril de 2018 